
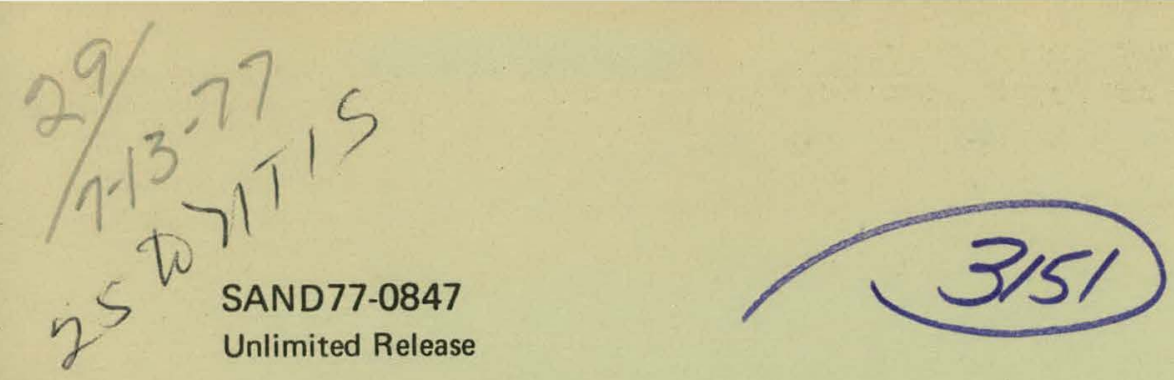

\title{
Computer Representation of Part Geometry
}

Richard H. Stark

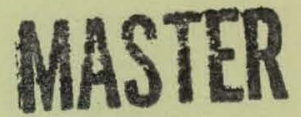

Prepared by Sandia Laboratories, Albuquerque, New Mexico 87115

and Livermore, California 94550 for the United States Energy Research

and Development Administration under Contract AT (29-1)-789

Printed June 1977 


\section{DISCLAIMER}

This report was prepared as an account of work sponsored by an agency of the United States Government. Neither the United States Government nor any agency Thereof, nor any of their employees, makes any warranty, express or implied, or assumes any legal liability or responsibility for the accuracy, completeness, or usefulness of any information, apparatus, product, or process disclosed, or represents that its use would not infringe privately owned rights. Reference herein to any specific commercial product, process, or service by trade name, trademark, manufacturer, or otherwise does not necessarily constitute or imply its endorsement, recommendation, or favoring by the United States Government or any agency thereof. The views and opinions of authors expressed herein do not necessarily state or reflect those of the United States Government or any agency thereof. 


\section{DISCLAIMER}

Portions of this document may be illegible in electronic image products. Images are produced from the best available original document. 
Issued by Sandia Laboratories, operated for the United States Energy Research \& Development Administration by Sandia Corporation.

\section{NOTICE}

This report was prepared as an account of work sponsored by the United States Government. Neither the United States nor the United States Energy Research \& Development Administration, nor any of their employees, nor any of their contractors, subcontractors, or their employees, makes any warranty, express or implied, or assumes any legal liability or responsibility for the accuracy, completeness or usefulness of any information, apparatus, product or process clisclosed, or represents that its use would not infringe privately owned rights.

Printed in the United States of America

Available from

National Technical Information Service

U. S, Department of Commerce.

5285 Port Royal Road

Springfield, VA 22161

Price: Printed Copy $\$ 4.00$; Microfiche $\$ 3.00$ 


\section{PAGES 1 to 2 WERE INTENTIONALLY LEFT BLANK}


SAND77-0847

Un limited Release

Printed June 1977

\title{
COMPUTER REPRESENTATION OF PART GEOMETRY
}

Richard H. Stark

Computer Aids Systems Development Division 9624

Sandia Laboratories

Albuquerque, New Mexico 87115

\begin{abstract}
ABST TRACT
A data structure defined in this paper is found to be flexible and economical for representing lie shapes of homogeneous solids. Its purpose is to support the activities of design, drafting, manufacture, and inspection in the manufacturing process. No attention is given to the problems of computational geometry which are inherent in all applications of such a representation.
\end{abstract}

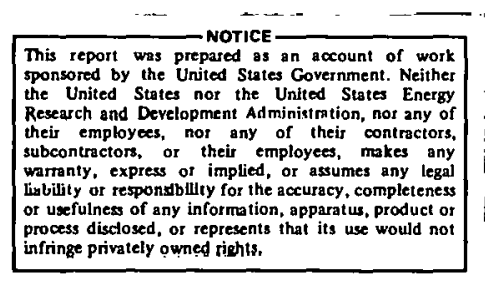




\section{CONTENTS}

Section

$\underline{\text { Page }}$

I. INTRODUCTION 7

I I. THE INGREDIENTS OF A REPRESENTATION. 9

III. : SPECIFICATION OF THE MODEL 13

IV. THE.STRUCTURE OF PIECES 14

$\begin{array}{lll}\text { V. SPECIFICATION OF A PIECE } & 17\end{array}$

VI. THE UPDATING OF FACES IN A SURFACE 19

VII. UPDATING PROCEDURES 28

VIII. CONCLUSIONS 31

IX. : BIBLIOGRAPHY $\quad \cdots \quad 31$ 
FIGURES

Figure

Page

1 A finite sequence of solids

8

2 A model requiring precise definition of face

3 A simple model and the boundary-face

ring for its top

4 Some primitives and their

: boundary-face rings

5. Two primitives and possible assignments of their faces to surfaces along with a sketch of the resultant shape from one of them

6 A face splits in two. New faces are created. A face edge is split in the process

7 . A new face is created in the same surface as a face that is modified. Its outward normals are directed oppositely to those of the face which is modified

8 An existing face is extended

9. A model edge splits a piece edge

10 Two holes in a model face are joined to make one

11 Two examples of removal from a model face by a piece face. The letter $M$ denotes model boundary, while $P$ denotecs piece boundary

12 Piece face exterior to model face but adjacent to it along a part of its boundary. The two faces have outward normals oppositely directed.

13. Isolating the bounding edges of an intersection of the piece face and a model face

14 Selecting a first candidate for relinking 


\section{INTRODUCTION}

A fundamental problem in machine support of the design and manufacture of machine parts is to establish an adequate internal description of the part geometry. It must facilitate the design process, support queries about physical properties, be adequate for generation of display tube or hard copy drawings, and be natural for the calculation of machine tool path. It has $i$ ts place in inspection and quality control after manufacture is complete. This report proposes a data structure for holding such a description and a process for generating its contents by successive approximations. It is based on insights provided by studying the work of Braid at Cambridge University $[1,2]$, Voelcker and associates at the University of Rochester [3], and on numerous conversations with Jim Graham, Dennis 0liver, and Don Peterson of Sandia Laboratories at Albuquerque.

\section{The Overall Concept}

We are concerned here only with homogeneous solids. We conceive any solid as being the limit (last of) a finite sequence of increasingly complex solids: The inilid solid has an elementary shape such as a rectangular parallelopiped. Each solid after the first is obtained by removing from its predecessor a "simple piece" of material for which a description is saved. Such a sequence is shown in Figure 1. If one is 
striving for a machinable reality, he may require that any piece removed have at least one face partially coincident with a face of the solid it modifies.
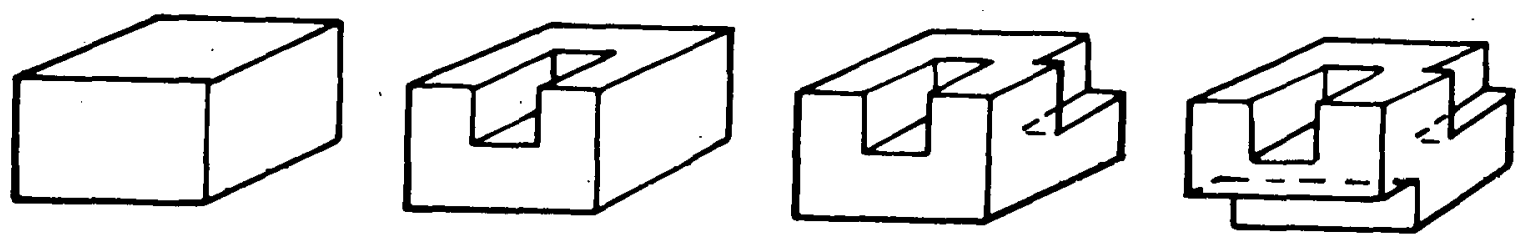

Figure 1. A finite sequence of solids

If such a process is to be effective for design, the following. are necessary:

1. The starting shape must be easy to describe.

2. The simple pieces to be removed must he pasily specified.

3. Updating the internal description from one solid to its successor must be a feasible machine program.

4. The internal representation must be sufficiently compact for available facilities.

An adequate data structure will also provide the items essential to generating display information and the data for numerical control of machines.

This report concentrates on items 1 . through 4 . The adequacy of the geometric representation for other functions can be judged in a broad way by the nature of the information kept. 


\section{THE INGREDIENTS OF A REPRESENTATION}

Throughout this report we shall refer to the description of the solid as a model and to the portions removed as pieces. We conceive of the shape of the model as being determined by a rigid skin of negligible thickness which has an inside consisting only of material of the solid it describes and an outside which is empty. We have avoided using the words face and surface in this statement since they will be defined in a special way for use throughout this report.

A simple surface is the range in three space of a continuous. mapping

$$
x=X(u, v), \quad y=Y(u, v), \quad z=Z(u, v)
$$

$$
\begin{aligned}
& u_{0} \leq u \leq u_{\text {MAX }} \\
& v_{0} \leq v \leq v_{\text {MAX }}
\end{aligned}
$$

from a rectangle in $(u, v)$ parameter space.

A patchwork surface is a simple surface in which the function of $X, Y, Z$ are defined over rectangles of the domain as

$$
x=x_{i j}(u, v), \quad y=y_{i j}(u, v), \quad z=z_{1 j}(u, v), \quad \begin{aligned}
& u_{i-1} \leq u \leq u_{i} \\
& v_{j-1} \leq v \leq v_{j}
\end{aligned}
$$

for $\underline{l} \leq i \leq I, \underline{l} \leq j \leq \mathrm{J}$. Note that $\dot{u}_{I}=u_{\text {MAX }}$ and $v_{J}=v_{\text {MAX }}$.

A smooth surface is a simple surface for which the direction cosines of the normal are continuous functions of the space coordinates $x, y$, and $z$.

Unless otherwise specified, when we speak of surface without using modifiers, we are assuming it to be smooth and permitting it to be a patchwork surface. 
Let $S$ be a surface and let there be a region of the skin of the model which coincides with this surface. We call such a region a face of the model in $S$ if

1. It is a maximal region which is the closure of its interior points (i.e., any point of the region has interior points of the region arbitrarily close. The points on the edge between $M$ and $N$ of Figure 2, for example, are not in a face containing $A B K J$ since they are remote from interior points of $A B K J)$.

2. It is connected (i.e., there is a path in the region connecting any two of $i$ ts points. The points $A$ and $F$ of Figure 2, for example, are not in the same face with respect to a plane containing $A B K J$ and $E F Q P$ since any curve connecting them in that surface was broken by 1).

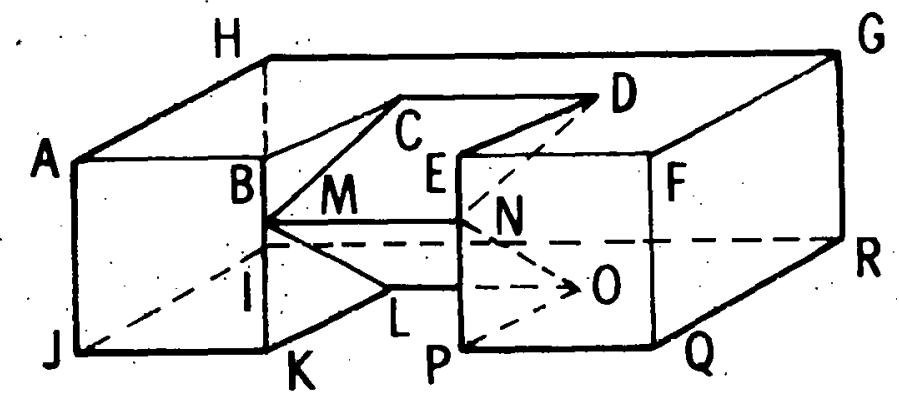

Figure 2. A model requiring precise definition of face

Given this definition of a face in a surface, we require that any model be such that we can define a finite number of surfaces which determine a finite number of faces such that the union of the faces contains all. points in the skin of the model and further such that no two of the given surfaces contain the same face. 
If two faces intersect along a curve, each is called a bounding face of the other. Their intersection determines a portion of the boundary of each. Two bounding faces for a given face determine two curves of intersection. If these curves intersect, they determine points on the boundary called vertices. That portion of a boundary contained between two consecutive vertices is called an edge. An intersection qualifies as an edge if it is a closed curve entirely in the skin even if it has no vertices. By this definition, vertices may occur at smooth transitions as at the intersection of the oval and straight edges of a stadium. They may also determine two or more distinct edges in the bourdary between two faces as in the boundary of the top face by the front face in Figure 3 .

Let a trihedral of mutually orthogonal vectors known as face, normal and edge vectors be associated with any point (not a vertex) on an edge. The face vector $f$ lies in the face, the normal vector $n$ is directed as the outward normals of the face (as determined at points in the face near the edge) and the edge vector $e$ is tangent to the edge. For a model edge trihedral, we choose a direction for the e-vector (also called edge direction and direction for boundary traversal) to be that of the extended right-hand thumb when curling the fingers of the hand describes a rotation of $90^{\circ}$ from face vector into outward normal. By contrast, for a piece edge trihedral, we direct the e-vector according to the thumb and fingers of the left hand for such a rotation. 
If one orders vectors by their alphabetic names, he finds these directions consistent with usual concepts of 1 eft and right-hand coordinate systems. It will be important to note that reversal of one vector in a piece edge trihedral changes its orientation to that of a model edge trihedral.

A boundary-face ring for a face, whether of the model or of a piece, is a circular list of bounding-face names ordered as they would be encountered in a traversal of a closed path in the boundary in the direction of its edges.

Note 1. A face with one or more holes in it would have a boundary-face ring for sach closed path in the boundary.

Note 2. Depending on the way it was formed, an hourglass region ( $\square$ ) in which a surface coincides with the skin of a model may be represented as one face or two. More complex examples of faces which have only vertices in common may likewise be consolidated into one. There is no difference in the models represented and the selection of the way to go can be made on implementation and efficiency considerations.

Example

In Figure $3 a$ we name a plane face by a string of three letters which are labels of vertices in its boundary. A boundary-face ring for $A B D$ with pointers to corresponding names of edges is shown in Figure $3 b$. 

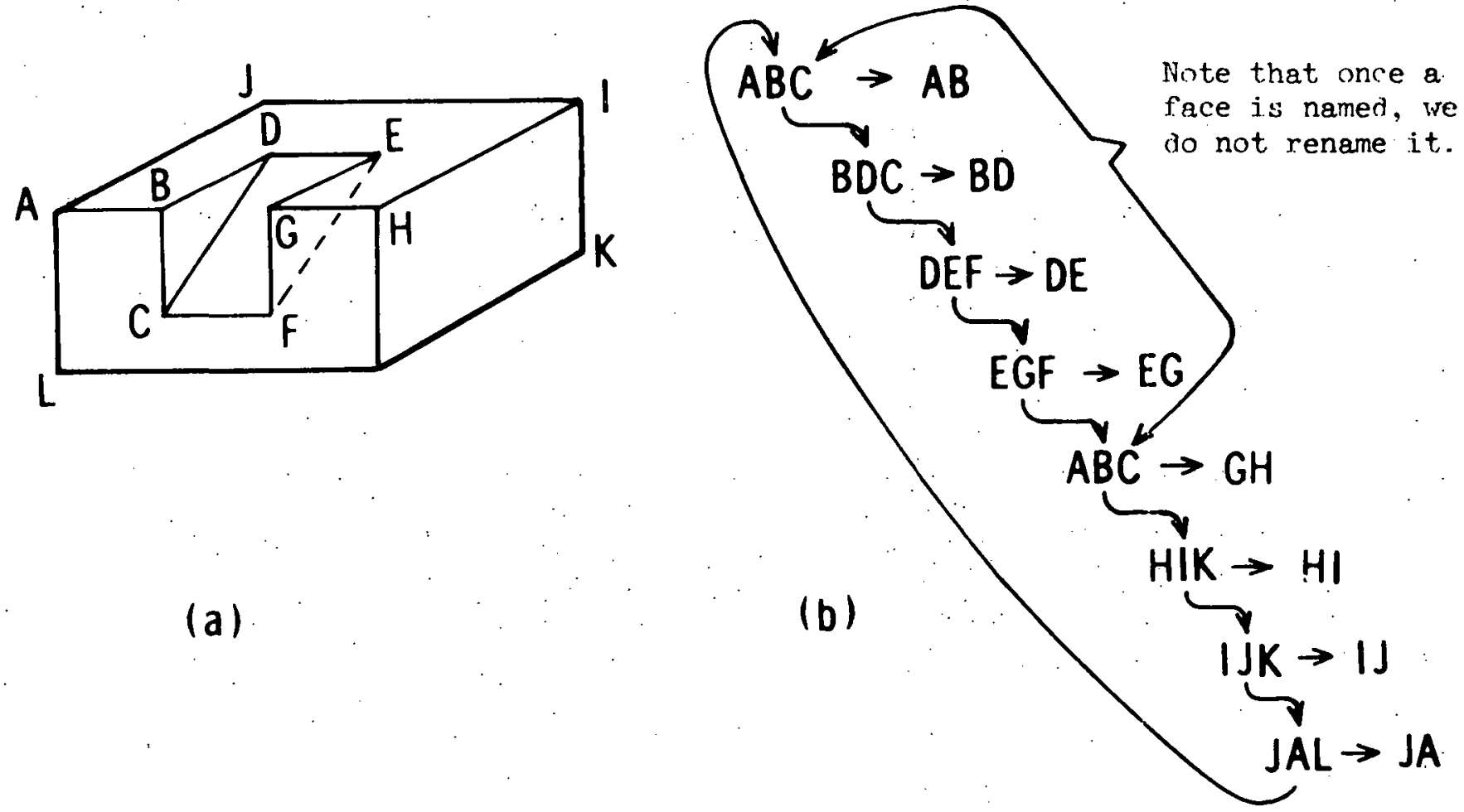

Figure 3. A simple model and the boundary-face ring for its top

\section{SPECIFICATION OF THE MODEL}

The description of the model contains the following:

1. A list of names of surfaces which are used in defining faces of the model with pointers to their definitions.

2. A list of names of faces in the model with a pointer to the name of the surface in which the face lies and a pointer to its list of boundary-face rings.

3. A record of the initial solid and the sequence of material removals which formed the model. (While this information may seem redundant, it is the most useful form of the description for some analyses.) 
Pieces to be removed in one operation are limited in complexity just to make the updating of the model convenient and economical. A library of partially defined pieces (called primitives) will be available. The key information provided for these basic pieces is a ring of names of bounding surfaces for each of its faces.

Defining the piece amounts to establishing a pointer for each face name to the name of a surface in the model which contains the face. If surfaces need to be added to the model description before this can be done, that is a preliminary step. The boundary-face ring determines the inside of the piece face. The edges which are associated with bounding faces can be calculated from surface intersections. Under no circumstances is the piece to be so defined that it occupies any volume outside the model it is to modify.

There are certain logical restrictions which a piece must satisfy and others which are used to simplify the problem. The restrictions currently contemplated are

1. The piece has no cavities (its complement is connected).

2. There must be no holes in a face of a piece. Even so, there may be more than one boundary-face ring as shown in the CAN primitive below. If there are, they must be vertex disjoint. 
3. If $S$ is a surface which already contains a face in the model and $F$ is a face of the piece assigned to $S$, then the piece cannot remove material from both sides of $S$ within the boundary of $F$.

Although the restrictions given above will permit quite complex pieces, the intent is that these be simple enough to facilitate calculation of mass properties and, even more important, to simplify the input needed to describe the model. Some simple concepts about piece primitives are illustrated in Figures 4 and 5 .

NAME OF

PRIMITIVE

CAN

$\operatorname{CONE}$

4.-FACE

\section{SKETCH}
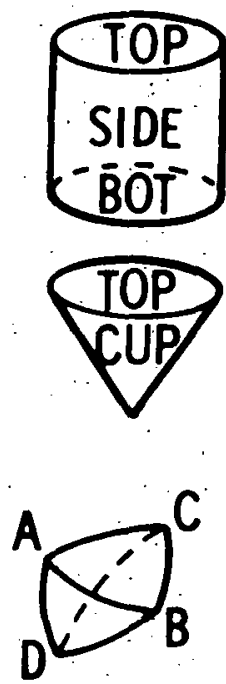

BOUNDARY FACE RINGS

NAME RING I : RING 2

TOP SIDE

B OT.

SIDE

SIDE

TOP

B.OT

$\begin{array}{ll}\text { TOP } & \text { CUP } \\ \text { CUP } & \text { TOP }\end{array}$

$A B C$

$B A D$

$B D C$

DAC

$B A D \cdots B D C$

$A B C$

$D A C$

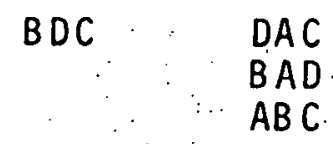

DAC

$A B C$

$B A D$

$B D C$

Figure 4. Some primitives and their boundary-face rings 


\section{PRIMITIVE}

CAN

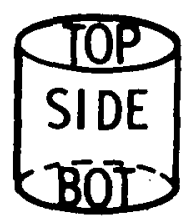

FACE

TOP

B OT

SIDE
SURFACE INTO WHICH ASS I GNED

Plane with direction numbers $(0,0,1)$ and at distance from origin equal to 5 .

Plane with direction numbers $(0,1,1)$ and distance from origin equal to 3 .

Cylinder with generatirix lire ection numbers $(0,0,1)$;

directrix is circle of radius 5 with conter at $(0,0,0)$ in pldile normal to generatrix

\section{7-FACE}

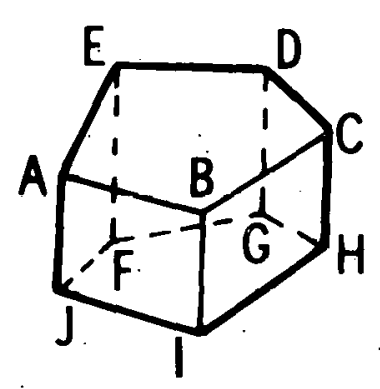

AEFJ

IBAJ

$Y=0$

$x^{2}+y^{2}=4$

HCB I

$Y=0$

GDCH

$X+Y=4$

FEDG

BCDEA

IHGFJ

$Y-X=4$

$z=5$

$Z=0$

Resultant shape from 7-FACE example

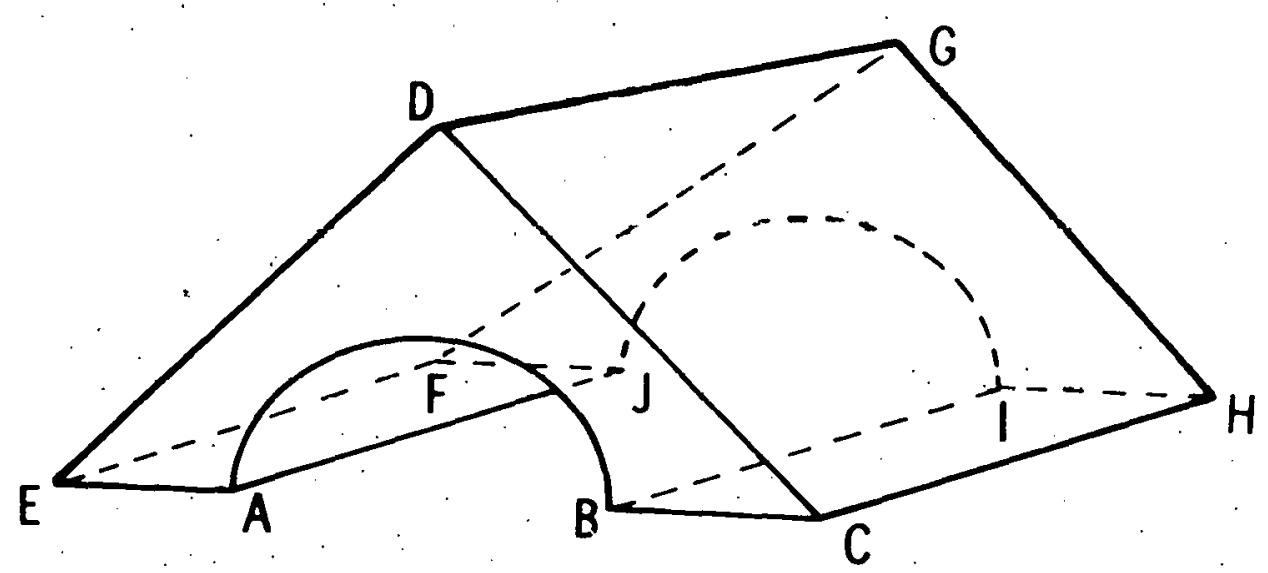

Figure 5. Two primitives and possible assignments of their faces to surfaces along with a sketch of the resultant shape from one of them 


\section{SPECIFICATION OF A PIECE}

From the examples above, it should be clear that the specification of a piece consists of

1. Selection of a primitive with the proper boundaryface ring structure:

2. Definition of surfaces which are required to place the piece but are not currently a part of the model specification.

3. A pointer to a model surface from every face in the primitive.

Some Examples of Updating the Model

Three dimensional geometry permits a multitude of special cases, and it will pay to develop some intuition by inspecting examples as given in Figures 6 through 10 before stating the arguments. Plane faces are assumed for sketch purposes. They are not essential.

MODEL

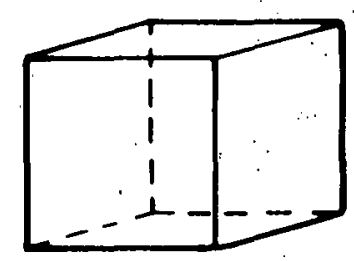

PIECE

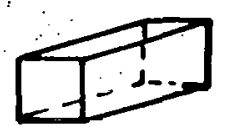

UPDATED MDDEL

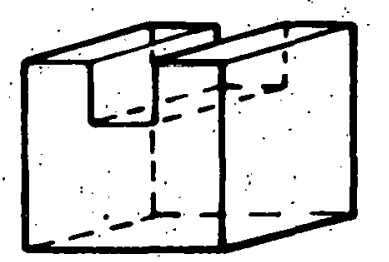

Figure 6. A face splits into two. New faces are created. A face edge is split in the process. 


\section{MODEL}

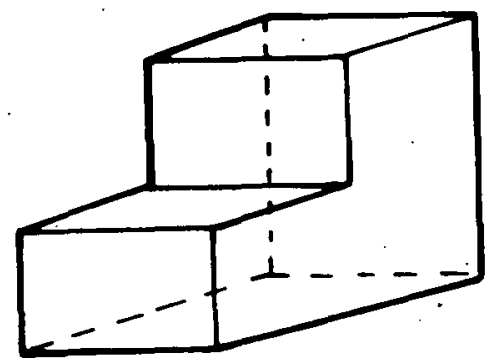

PIECE

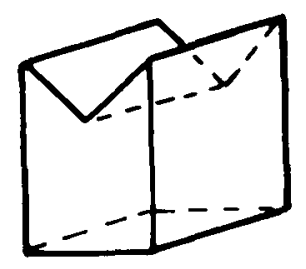

UPDATED MODEL

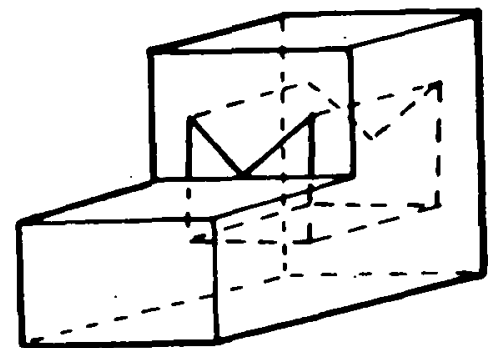

Figure 7. A new face is created in the same surface as a face that is modified. Its outward normals are directed oppositely to those of the tace which is modified.
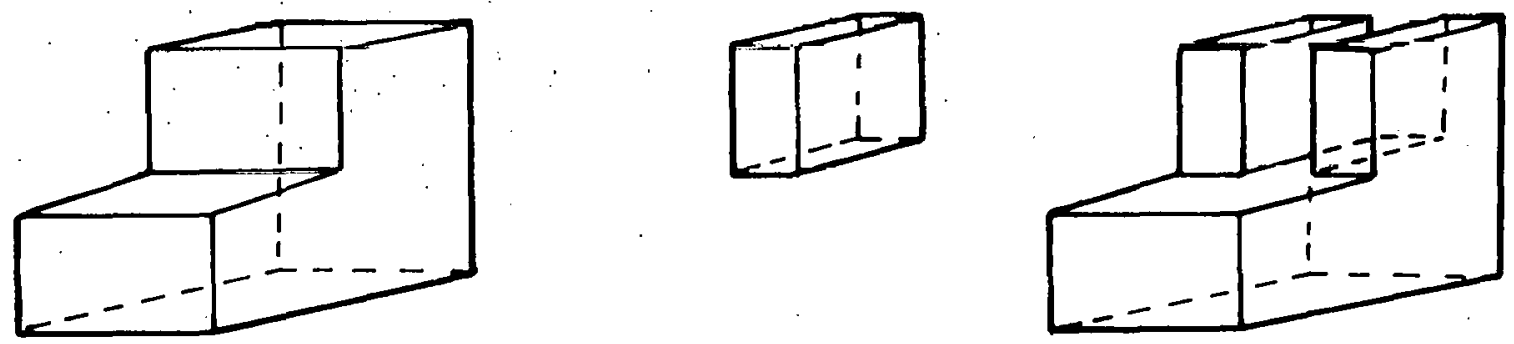

Figure 8. An existing face is extended
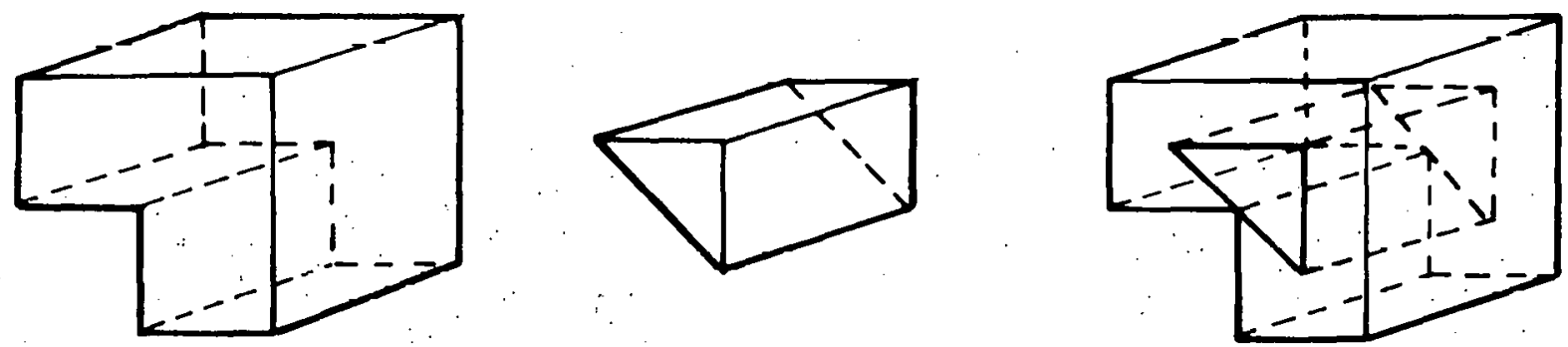

Figure 9. A model edge splits a piece edge.
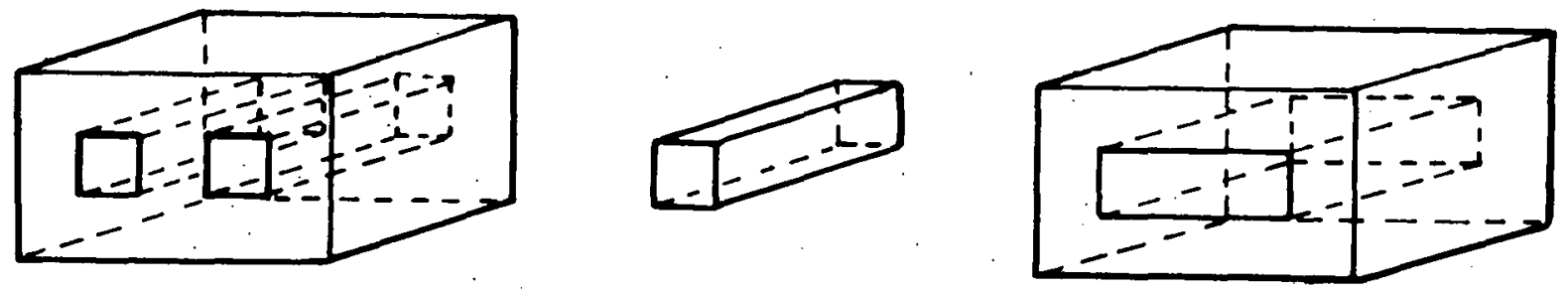

Figure 10. Two holes in a model face are joined to make one. 
Boundary-face rings pertain only to faces. In considering how these are modified with the application of a piece, we may limit our analys is to the faces in one surface at a time and within that surface to the effects of one piece face at a time.

The first step in application of a piece is to insert new vertices in the face boundary of the model and in the face boundary of the piece to ensure that

1. If two edges have more than endpoints in common, they coincide.

2. If two edges do not coincide, their representations are adequate to show this.

The lemmas and theorem of this section may be applied so that by making adjustment at one vertex at a time over all the vertices in the piece face and in the model faces touched by the piece face, we may update the set of model faces in the surface. Action at one vertex may be taken without any respect to action taken at other vertices.

We find it convenient to alternate between elements of a boundary-face ring and the edges which they determine. Since there is a one-to-one correspondence, this should cause no difficulty. 
$\underline{\text { Lemma } 1}$

If the boundary. of a piece face has no points in common with any model face, then the piece face will with reversal of $i$ ts outward normal become a face of the updated model, and its boundary-face rings will be the boundary-face rings of the new model face.

Proor: Sillce llo puint of the plece face is in a face of the model, the piece face must be entirely interior to the model as the model was prior to updating. The piece removes material up to its faces. The outward normal of the piece face will be opposite to that of the model face it creates. Reversing the direction of the outward normal will change the piece edge trinedral to a model edge trihedral and leave the face vector in the model face. Thus we find the boundary-face ring for the piece is correct for the new model face without change.

Lemma 2

If a piece face has no points exterior to a given model face, then the model face(s) resulting from the update will be obtained by deleting from the original model face its intersection with the piece face. The boundary-face rings of an updated model face are obtained from those of model face and piece face by relinking where the boundaries join or separate. Where a piece face edge is just the reverse of a model face edge, these edges will both be dropped from boundary-face rings. 
Proof: Consider a segment of piece face boundary which either never touches a model face boundary or does so only at its extremes and in either case is distinct from the model face boundary on its interior. That segment separates material remaining in a model face from void created by removal. Its boundary faces and edges (possibly reversed in direction) will be in the boundary-face ring of the model face. Since the piece face deletes from the model face, its outward normal is an outward normal to the model face. Reversing the face vector of a piece edge trihedral yields a model edge trihedral with face vector in the model face and edge vector directed along the new model face edge. Therefore these piece face edges become model face edges, and the selected segment of the piece face boundary will be in the boundary-face ring of the updated model face. If the piece segment boundary does not touch the model face boundary, that segment will constitute a new boundary-face ring for the model face.

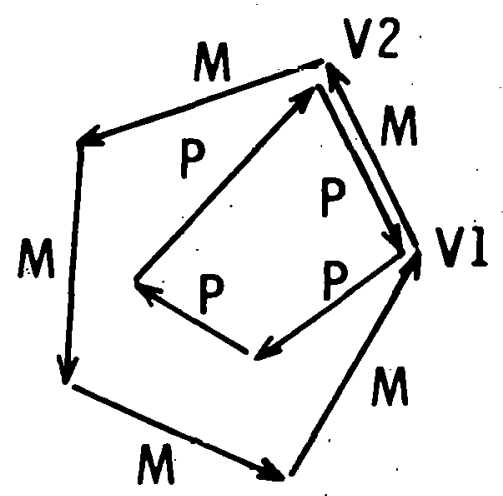

(a)

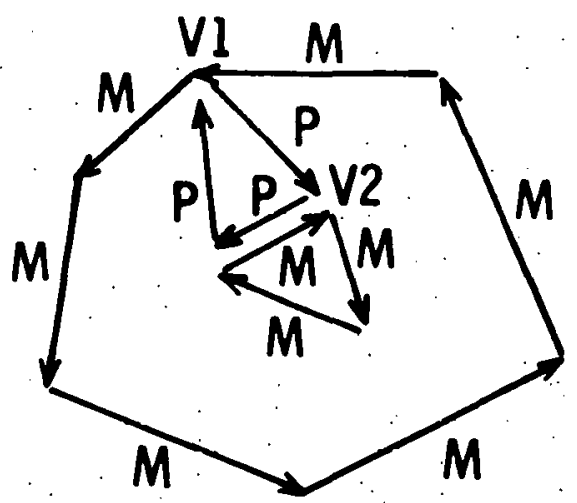

(b)

Figure 11. Two examples of removal from a model face by a piece face. The letter $M$ denotes model boundary, while $P$ denotes piece boundary. 
If the piece face segment meets the model face boundary at one extreme, it must at the other as well, for its boundary is closed and al ready has one extreme on the model face boundary. Let $v 1$ be the extreme at which the segment separates from the model faceboundary and $v 2$ be the extreme at which it rejoins. These two could be the same vertex or could even be on disjoint boundary curves as in Figure 1lb. Each vertex will have two piece edges incident, one with its head at the vertex called the entering piece edge and one with its tail at the vertex called the exiting piece edge. Entering and exiting model edges are similarly defined. At $V l$, we make the exiting piece face edge the successor to the entering model face edge. At the V2 end of the segment, we make the exiting model face edge the successor to the entering piece face edge. This relinking is done for every segment of the piece face boundary which touches the model face boundary at its extremes and is distinct from it on its interior. On completion, we have

1. Every edge of the $\left[\begin{array}{l}\text { model } \\ \text { piece }\end{array}\right]$ face which is not the reverse of a $\left[\begin{array}{l}\text { piece } \\ \text { model }\end{array}\right]$ face edge is in a circular list.

2. Every edge in each circular list is directed properly to qualify as a model face edge.

Thus, the resulting rings constitute the boundary of the updated model face. It remains to be noted that when a piece face edge is the reverse of a model face edge, the two are automatically lost from the boundary-face rings. 
Lemma 3

If a piece face is exterior to all model faces in its surface, then the updated model can be determined without any reference to faces which have their outward normals similarly directed (with respect to their common surface) to those of the piece. Those model faces which have their outward normals opposite in orientation to those of the piece need to be recognized only where they are adjacent along an edge to a piece face.

Proof: Two faces are defined to be distinct if their outward normals are oppositely directed, even if in the same surface and touching: The piece face normals are opposite to those of the new face it creates exterior to the original faces. Thus, unless the piece face normals oppose those of the model face, there can be no interaction. For the case that the piece face outward normals are opposed to those of a model face; there can surely be no interaction if they have no points in common. If the piece face and a model face touch at a vertex but not along edges incident to that vertex, it is arbitrary whether the model face area created by the piece is merged with the original or not. The model represents the same solid and has the same edge assortment in ei ther case. The choice whether to relink in order to merge faces at such a common vertex is left to implementation. The relinking required when the piece face and model face have edges in common and outward normals opposed is covered in Lemma 4 . 


\section{Lemma 4}

If a piece face is exterior to all model faces but adjacent to one or more model faces along respective edge segments, and if the piece has outward normals oppositely directed to those of the model faces (relative to the surface in which they all lie) then the updated model face is the union of piece face and edgeadjacent model faces. The boundary-face rings of the updated model face will be obtained from those of piece face and all such edge-adjacent model faces by relinking where the boundaries join or separate.

Proof: According to Lemma 3, we are encitled to ignore all kinds of contact between the piece face and model faces to which it is exterior except for the edge adjacencies of a piece face with model faces having outward normals oppositely directed. This situation is represented in Figure 12 .

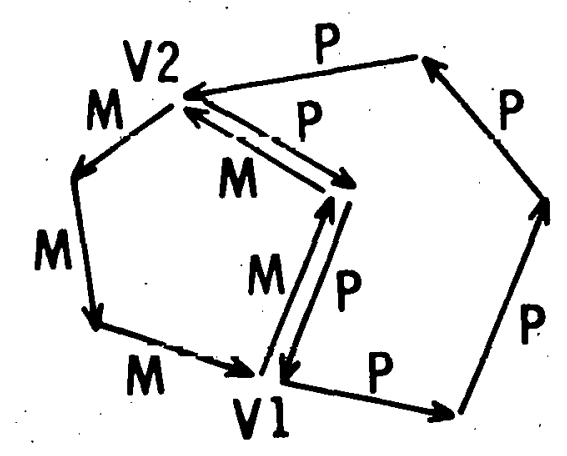

Figure 12. Piece face exterior to model face but adjacent to it along a part of its boundary. The two faces have outward normals oppositely directed. 
The model face created by removing the piece from the model will have outward normals directed to the same side of the surface as the originals. Any segment of the piece boundary which has vertices in common with a model face only at extremes and which is distinct from the model face on its interior must be part of the updated face boundary. The face vector of the piece edge trihedral lies in that new face and reversing its normal to fit the outward normal of the model face gives a model edge trihedral which qualifies the piece edge as a model face edge. Thus the portion of the boundary-face ring of the piece corresponding to this segment must be in the ring for the model face. Let the segment be from $V 1$ to $V 2$. We make the exiting $\left[\begin{array}{c}\text { piece } \\ \text { model }\end{array}\right]$ face edge the successor to the entering $\left[\begin{array}{l}m o d e l \\ p i e c e\end{array}\right]$ face edge at $\left[\begin{array}{l}V l \\ V 2\end{array}\right]$ for every such segment. Then the conditions 1. and 2. of Lemma 2 are met. When this has been done for each qualifying model face, the resulting rings will delineate the boundary of the updated composite face. Note that the faces giving edges along which original model faces and the piece face were adjacent are automatically dropped from the boundary-face rings.

\section{Theorem}

Let the set of model faces in a surface be $\left[M_{1}, M_{2}, \ldots, M_{k}\right]$ and the piece face applied to this surface be $P$. Denote the inter-: section of $P$ with $M_{j}$ by $I_{j}(j=1, \ldots, k)$. Then the model faces in the updated mode $1 \underset{k}{\text { will }} 1$ be constructed from $M_{1}-I_{1}, M_{2}-I_{2}, \ldots, M_{k}-I_{k}$ and the residual $P-U, I_{j=1}$. The boundary face rings of the resultant model faces are obtained from those of $P$ and of the original faces 
by relinking where piece face boundaries join or separate from model face boundaries:

Proof: Let $M_{j}$ be a model face which is actually penetrated by the piece face. Let $V$ l. be a vertex of $M_{j}$ such that

1. the entering piece face edge lies outside $M_{j}$ and is not on the boundary of $M_{j}$; and

2. a piece boundary traversal starting at $V 1$ encounters only edges along the boundary of $M_{J}$ until reaching a vertex $V 2$ at which the exiting piece face edge departs from the boundary and $l i$ es in $M_{j}$ (see Figure 13).

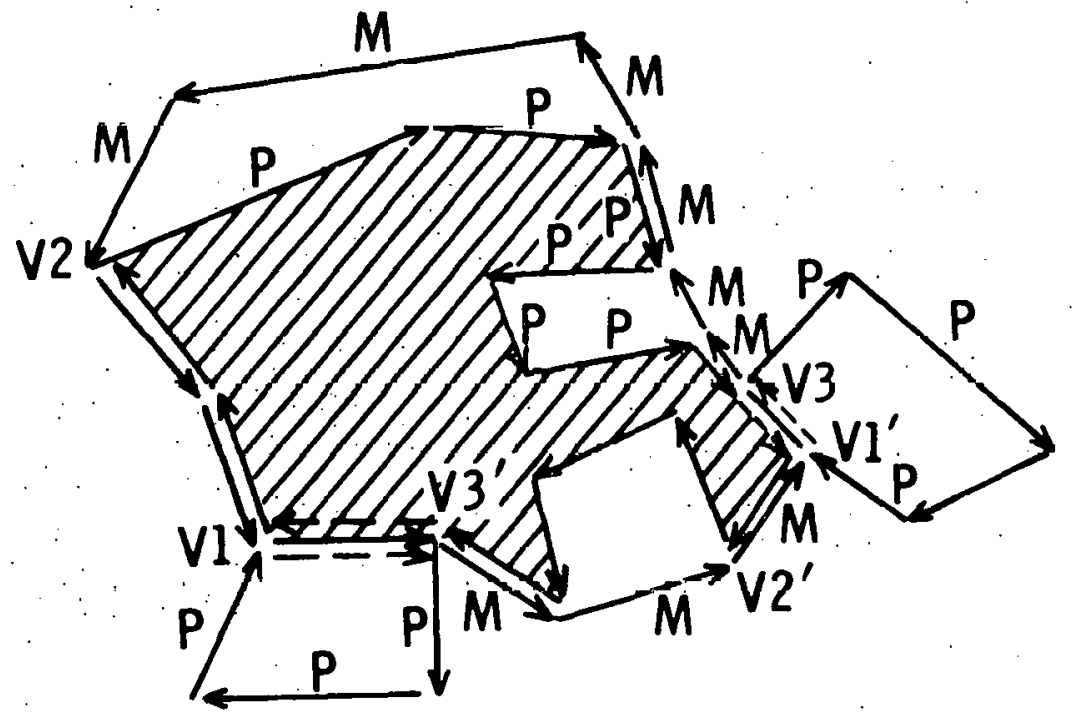

Figure 13. Isolating the bounding edges of an intersection of the piece face and a model face. 
Let $V 3$ be the first vertex of $M_{j}$ reached in a piece face edge traversal beginning at $V 2$ for which the exiting piece face edge lies exterior to $M_{j}$ and not on $i$ ts boundary. As seen in Figure 13, there may be more than one such path vi to V3. All such segments of the piece face boundary are in the boundary of $I_{j}$. We must now complete that boundary: Let the set of vertices of type $V 1$ and $V 3$ be denoted as $\gamma l$ and $\gamma 3$ respectively. We select a member $v 3^{(1)}$ of $\gamma 3$ and traverse the piece face boundary until arriving at the first vertex which is a member of $\gamma l$. Denote this vertex as $v_{l}(1)$. These two vertices determine two segments along the boundary of $M_{j}$, one directed from $v_{3}(1)$ to ${ }_{V}{ }^{(1)}$. Which we include as one of the boundary segments of $I_{j}$ and one from $v_{1}(1)$ to $V_{3}(1)$ which closes the portion of the piece face exterior to $M_{j}$. Such segments are shown as dashed lines in Figure 13. Let this construction be continued for pairs of segments corresponding to selections $v_{3}(i)$ and $v_{1}(i) \quad(i=2,3, \ldots)$. until the sets $\gamma 3$ and $\gamma l$ are exhausted. The segments we have chosen to form the boundary of $I_{j}$ form a closed curve. They enclose all that could be in $I_{j}$ ard no more. Thus we have constructed a boundary face ring for $I_{j}$. Assuming that no other such constructions have previously been made, the boundary-face rings for $P-I_{j}$ are completed by replacing the piece edge segment from $V l^{(i)}$ to $V 3^{(i)}$ by the boundary segment constructed above for each such vertex pair. This construction can be done for each non-empty $I_{j}$. What it accomplishes is dividing the piece face into subfaces, each of which is entirely within a model 
face or else exterior to all model faces. We can then apply Lemma 2 to the $I_{j}$ subfaces sequentially and then Lemma 4 to the residual which is exterior to all model faces in the surface.

Even though it may seem we have introduced new sequences of edges into the boundary face rings, the relinking rules remain as indicated. The $v_{1}(i)$ to $v_{3}(i)$ segments along the $M_{j}$ boundary are identical to the $M_{j}$ boundary segment between those vertices since outward normals coincide, and their face vectors are oppositely directed. Thus, we can make the exiting $M_{j}$ edge at $v_{l}(i)$ the successor to the entering piece face edge. and the exiting piece face edge at $v 3^{(i)}$ the successor to the entering $M_{j}$ edge. If one of the vertices has no incident edge adjacency of piece face and $M_{j}$ edge (e.g.. if V2 coincides with V1 in Figure 13), then each of these relinkings occurs at that vertex.

\section{UPDATING PROCEDURES}

The only vertices which need to be considered are those on the piece face boundary as reconstituted at the beginning of $V I$ by addition of vertices where necessary to assure that two edges which do not coincide will intersect only at endpoints. 
Single Model Face Relinking Rules.

If a vertex has two piece face edges and exactly two model face edges incident, then if the exiting [ $\left.\begin{array}{c}\text { piece } \\ \text { model }\end{array}\right]$ face edge

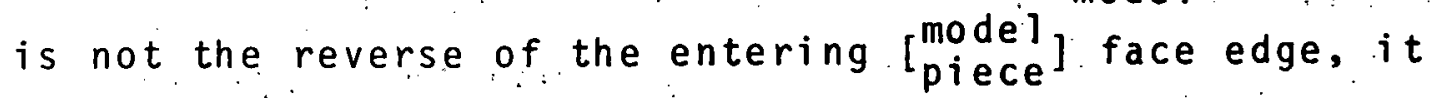
is made the successor in a boundary-face ring to that entering edge.

Multiple Model Face Relinking Rules.

Given a vertex at which as many as two model faces and one piece face have incident edges, the single model face rule is not determinate. We are concerned first with just those model faces which are penetrated by the piece face. All these must have outward normals oriented similarly to that of the piece.

We take the entering piece face edge as the first candidate for relinking. However, if it happens to be the reverse of an exiting edge of a model face $M_{j}$, we cancel both and replace the piece edge as a candidate by the entering edge for $M_{1}$. In either case, we have an entering edge $I_{1}$ as a starting point. Four possible cases are shown in Figure 14.

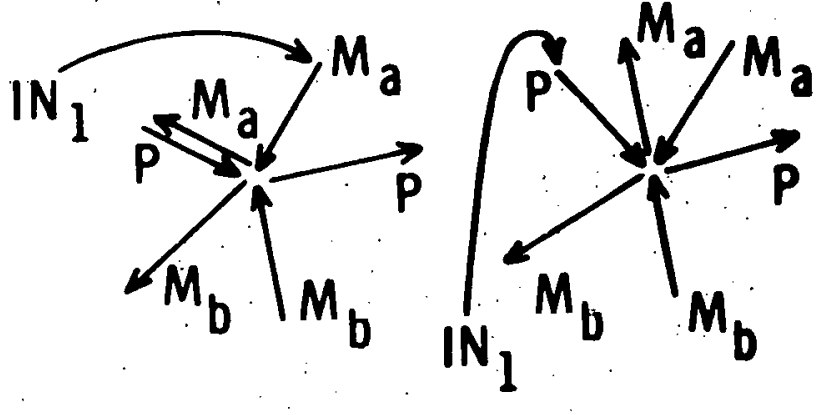

(a) (b)

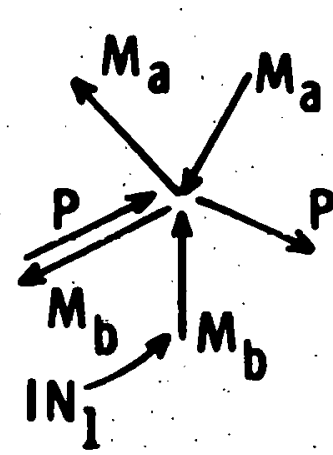

(c)

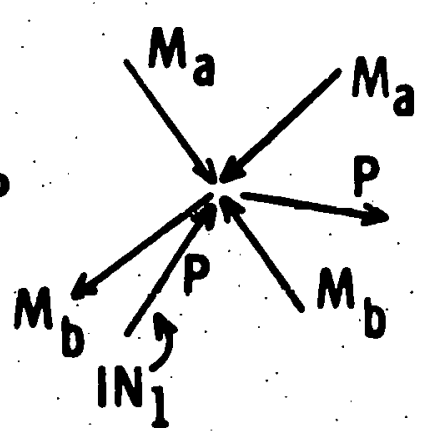

(d)

Figure 14. Selecting a first candidate for relinking. 
Before attempting to find a successor to $I_{1}$, we first determine how the edge relinking is to terminate. We take as initial candidate for the last relinked successor the exiting piece face edge. If it is the reverse of an entering edge of a model face $M_{k}$, then we cancel both and make the exiting edge of that face the last edge to be a relinked successor: call it OUTLAST

It is now appropriate to picture the edges to be relinked as those in the. "extended piece face" bounded by IN, and OUTLAST and containing the original piece face. Each entering edge finds its successor by the following rules:

1. The successor must be within the extended piece face.

2. The successor cannot be separated from the entering edge by another entering edge.

3. Relinking starts with $I_{1}$ and continues with each incoming edge which was freed by taking its successor for relinking; until the OUT LAST edge is relinked.

That these rules are adequate stems from the requirements that model faces having at most a vertex in common with the piece face are not modified and that model faces cannot overlap. 


\section{CONCLUSIONS}

This paper is intended only as a demonstration that there is a feasible way to represent the geometrical aspects of a large class of solids which are manufactured; one which is convenient for design, display and manufacture. It leaves open many questions about representation of surfaces and edge curves. We consider this to be an achievement, for by restricting the operations permitted with primitives, it provides a substantial relaxation of restrictions placed on shape in the investigations of Braid and Voe1cker.

\section{BIBBLIOGRAPHY}

Braid, I.C. Designing With Volumes Cantab. Press, Cambridge England 1973.

Braid, I. C. The synthes is of solids bounded by many faces. Comm. ACM 18,4 (April 1975), 209-216.

Voelcker, H. B.; Requicha, A.A.G.; Samuel, N..M. Part and Assemb ly Description Languages TM20a, Production Automation Project, University of Rochester. Nov. 1974: 
DISTRIBUTION:

Computer Science Dept.

New Mexico State University

Box 3CU

Las Cruces, NM 88003

Attn: J. Mack Adams (1)

Attn: R. H. Stark (5)

Lawrence Livermore Laboratory

P. 0. Box 808

Livermore, CA 94550

Attn: J. Jantzen (1)

Attn: J. L. Martin (1)

Attn: Robert Westbrook

Los Alamos Scientific Laboratory

P. 0 . Box 1663

Los Alamos, NM 87545

Attn: Gordon Anderson,

SD-DO, MS 472 (1)

Attn: Donald J. Lauer,

$$
W X-3 \text {, MS } 932 \text { (1) }
$$

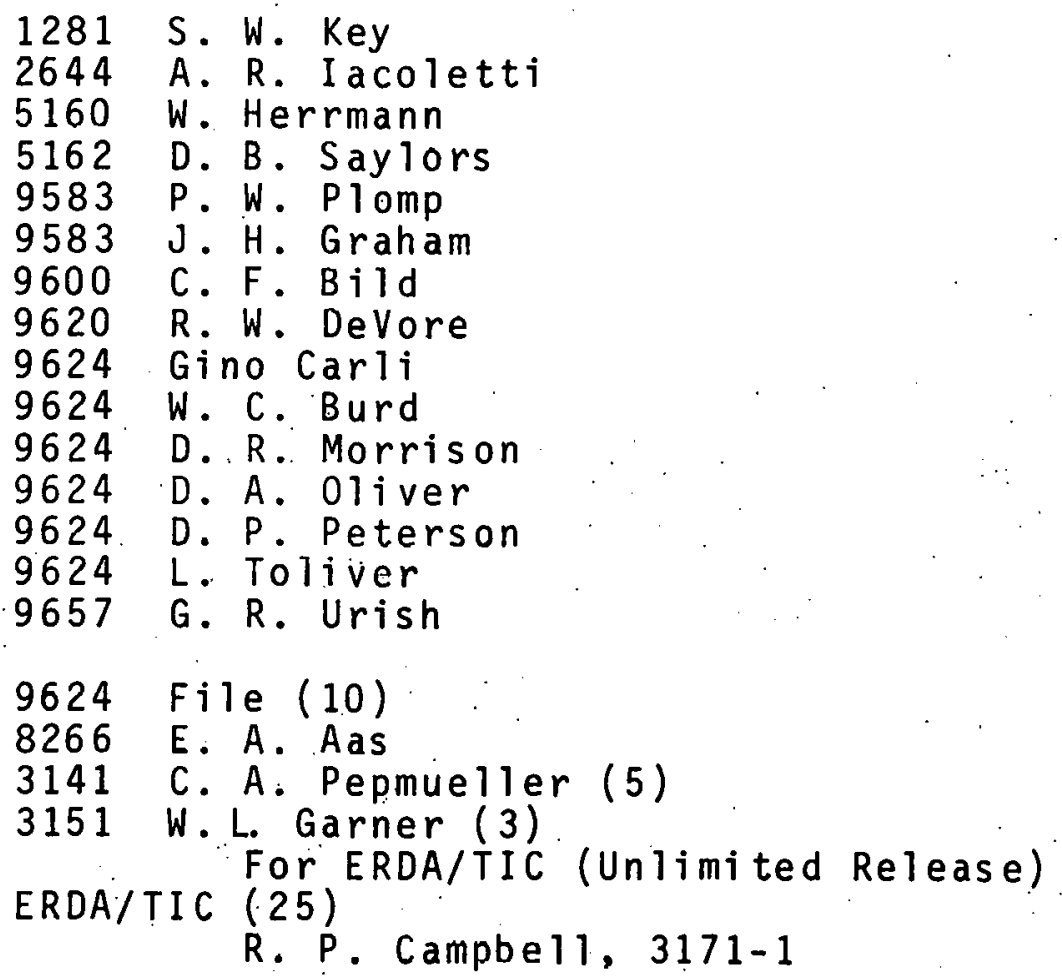

